\title{
Uniform connectedness and classical Gestalt principles of perceptual grouping
}

\author{
SHIHUI HAN \\ University of Science and Technology of China, Beijing, China \\ GLYN W. HUMPHREYS \\ University of Birmingham, Birmingham, England \\ and \\ LIN CHEN \\ University of Science and Technology of China, Beijing, China
}

\begin{abstract}
We assessed whether uniform connectedness (UC; Palmer \& Rock, 1994) operates prior to effects reflecting classical principles of grouping: proximity and similarity. In Experiments 1 and 2, reaction times to discriminate global letters ( $\mathrm{H}$ vs. $\mathrm{E}$ ), made up of small circles, were recorded. The small circles were respectively grouped by proximity, similarity of shapes, and by UC. The discrimination of stimuli grouped by similarity was slower than those grouped by proximity, and it was speeded up by the addition of UC. However, the discrimination of stimuli grouped by proximity was unaffected by connecting the local elements. In Experiment 3, similar results occurred in a task requiring discrimination of the orientation of grouped elements, except that the discrimination of stimuli grouped by UC was faster than that of those grouped by weak proximity. Experiment 4 further showed that subjects could respond to letters composed of discriminably separate local elements as fast as to those without separated local elements. The results suggest that grouping by similarity of shapes is perceived slower than grouping by UC, but grouping by proximity can be as fast and efficient as that by UC.
\end{abstract}

It is widely assumed that grouping of separated objects occurs early in visual processing. The function of perceptual grouping is to solve the problem of "what goes with what" and the differentiation of figure from ground (Rock, 1986). Many studies suggest that the visual field is preattentively segmented into separate figural units or objects through perceptual grouping, which are further processed by the operation of focal attention for identification (Duncan, 1984; Duncan \& Humphreys, 1989; Kahneman \& Henik, 1981; Kahneman \& Treisman, 1984; Moore \& Egeth, 1997; Neisser, 1967; but see Mack, Tang, Tuma, Kahn, \& Rock, 1992).

Perceptual grouping has typically been discussed in terms of the principles of grouping first described by the Gestalt psychologists (Koehler, 1928; Wertheimer, 1923). One of the principles of grouping is proximity, which states that spatially close objects tend to be grouped together. The principle of similarity claims that, all else being

This study was supported by the State Commission of Science and Technology and the National Foundation of Sciences, People's Republic of China, by the Human Frontier Science Program, and by the BBSRC (United Kingdom). The authors thank X. Zhang for development of computer software and F. Xiao for running Experiment 2. Correspondence should be addressed to $S$. Han, Beijing Lab of Cognitive Science, Graduate School, University of Science and Technology of China, 19A Yuquan Rd., P.O. Box 3908, Beijing 100039, People's Republic of China (e-mail: shhan@public2.bta.net.cn).

-Accepted by previous editor, Myron L. Braunstein equal, the most similar elements in the field tend to be grouped together. Two of the other Gestalt laws of grouping are common fate and good continuation. Common fate states that elements that move simultaneously in the same direction and with the same speed tend to be grouped together. Good continuation refers to the grouping of two or more contour regions into one unit on the basis of smooth continuation of contour from one region to another.

Although the Gestalt principles have been proposed for several decades, only a few studies have concerned the relationship between different principles of grouping. For example, Kurylo (1997) reported evidence showing that the processing time for grouping by proximity was shorter than that for grouping by alignment (i.e., good continuity). Some studies have been guided by the hypothesis of early topological perception (Chen, 1982), which assumes that a primitive function of the visual system is to encode the presence of topological differences in the image. For instance, Chen (1986) required subjects to report the horizontal or vertical organization of arrays of stimuli in which proximity and similarity provided conflicting grouping cues. He found that subjects reported groups on the basis of proximity and similarity of closure (one sort of topological property) when stimuli were displayed for a short duration. However, subjects responded in accordance with the similarity of local geometrical properties (e.g., orientation) of the elements when stimuli were presented for a long duration. Chen (1986) proposed that, with respect to the time dependence of perceptual grouping, proximity oc- 
A
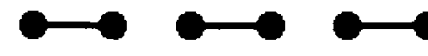

B

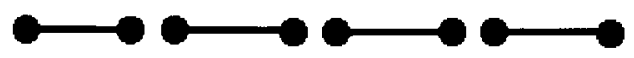

C

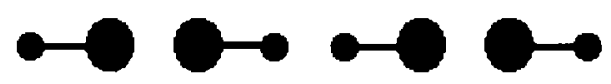

D

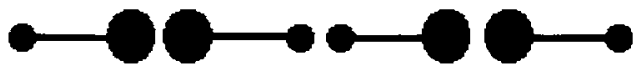

Figure 1. Illustration of uniform connectedness as a principle of perceptual grouping (A), its dominance over proximity (B), similarity in size (C), and both proximity and similarity (D). (From Palmer \& Rock, 1994.)

curs prior to similarity; similarity based on topological properties (e.g., similarity of closure) is perceived prior to similarity based on local geometrical properties (e.g., similarity of orientation). Ben-Av and Sagi's (1995) work has provided more evidence on this. Using a paradigm similar to that employed by Chen (1986), Beh-Av and Sagi found that proximity grouping was perceived much faster than grouping based on similarity of luminance and shape and that proximity dominated performance when the stimulus was available for a short time $(<100 \mathrm{msec})$. With increasing processing time, similarity grouping was found to dominate performance.

The different roles of proximity and similarity in perceptual grouping have also been found in the processing of Navon-type (Navon, 1977) compound stimuli (Han, Humphreys, \& Chen, in press). Han et al. compared the relative advantage of global and local properties of stimuli in conditions in which the compound stimuli were presented without or with background patterns. Similar to the observations of other researchers (Hughes, Layton, Baird, \& Lester, 1984; Luna, Marcos-Ruiz, \& Merino, 1995; Navon, 1977; Navon \& Norman, 1983), Han et al. found a global precedence effect when no background patterns were presented (i.e., subjects responded faster to global figures than to local figures, and global figures interfered with responses to local ones when the figures at the two levels were inconsistent). However, when proximity grouping was eliminated by embedding the compound stimuli in background patterns (so that grouping was forced to depend on the shape similarity of local figures), reaction times (RTs) to local figures were faster than or the same as those to global figures, and local interference over global figures also became stronger than the reverse. These results suggest that the advantage for global, compound figures depends on proximity grouping between local figures, and proximity dominates similarity of shapes for grouping local figures into a unitary global form. In comparison with proximity grouping, similarity grouping occurs at a later stage of perception.

Two possible new principles of perceptual grouping have been put forward in recent years by Palmer and Rock
(Palmer, 1992; Palmer \& Rock, 1994). One of them is called common region (Palmer, 1992), which states that, all else being equal, elements will be perceived as being grouped together if they are located within a common region of space (i.e., if they lie within a connected, homogeneously colored or textured region or within an enclosing contour). Palmer further argued that common region cannot be reduced to the effects of proximity, closure, or any other previously known factors. More recently, Palmer and Rock (1994) have proposed another new grouping principle, uniform connectedness (UC), which asserts that a connected region of uniform visual properties (e.g., luminance or lightness, color, texture, motion, and possibly other properties as well) strongly tends to be organized as a single perceptual unit. To demonstrate the existence of the UC principle and its relation with classical grouping principles, an illustration such as that in Figure 1 was used in Palmer and Rock's article. A horizontal array of black dots with the same distance between two adjacent items was segmented into separate groups by connecting two neighboring items with lines (Figure 1A). Thus, grouping two neighboring dots by connecting them seems to dominate grouping by other factors, such as proximity (Figure 1B), similarity (Figure 1C), or both proximity and similarity (Figure 1D). On the grounds of this demonstration, Palmer and Rock argued that the UC principle can overcome other classical grouping principles, such as proximity and similarity, and that $\mathrm{UC}$ is such a strong factor in perceptual organization that it occurs prior to other classical Gestalt principles of grouping and virtually defines primitive perceptual units at a very early stage.

The influence of UC on perceptual organization and visual selective attention has been supported by Kramer and Watson's (1996; Watson \& Kramer, 1999) recent work, which showed that RTs were faster when perceptual judgments involved two aspects of a single UC region than when they involved two different UC regions. They argued that this same-object effect produced by UC suggests that $\mathrm{UC}$ is crucial in defining the entities available for attention selection. Nevertheless, Palmer and Rock's (1994) argument for the relationship between UC and the classical Gestalt principles of grouping has not been tested systematically. Simple illustrations may conceal some differences between the new principles of grouping and classical principles of grouping, such as proximity and similarity, particularly if different groupings will dominate as a function of the time course of processing. To uncover the relationship between the possible new principles of grouping and the classical grouping laws, quantitative measures are essential.

The objective of the present study was to investigate the relationship between UC and grouping based on proximity and similarity. Palmer and Rock (1994) asserted that UC "can overcome the law of proximity" (p. 31). One possible reason for this may be that UC occurs earlier in time than proximity. Another possible reason is that both UC and proximity may occur at the same time, but physically connected elements form a better group than do physically close elements. We compared subjects' RTs to 
discriminate global letters made up of small circles. The circles composing a letter were grouped together according to different principles of grouping, such as proximity, similarity, or UC. We also recorded RTs to discriminate orientations of perceptual groups formed by similarity, proximity, or UC. The rationale is that, all else being equal, earlier or stronger grouping between the local elements should reduce RTs to discriminate both global letters (Experiments 1,2, and 4) and the orientations of perceptual groups (Experiment 3 ).

\section{EXPERIMENT 1}

Since grouping by proximity occurs earlier than grouping by similarity of shapes (Ben-Av \& Sagi, 1995; Chen, 1984, 1986; Han et al., in press), all else being equal, RTs to letters based on grouping by proximity should be faster than those to letters based on grouping by similarity of shapes. In terms of a similar rationale, if grouping based on UC occurs earlier than grouping based on proximity and similarity, RTs to letters constructed from connected elements should be faster than those to letters in which local elements are grouped by proximity or similarity. Differences between RTs to the letters grouped by $\mathrm{UC}$, proximity, and similarity may also reflect different time courses of perceptual grouping. Hence, we compared performance across time, as a function of the interval between the stimulus and a mask. There were four conditions for grouping small elements (circles) into a global letter: grouping by proximity, grouping by proximity and UC, grouping by similarity, and grouping by similarity and UC.

\section{Method}

Subjects. The subjects were 3 female and 14 male right-handed, paid volunteers (22-32 years of age) from the Graduate School of University of Science and Technology of China. All had normal or corrected-to-normal vision.

Apparatus. Data collection and stimulus presentation were controlled by a 386 personal computer. Stimuli were presented on a 12 in. color monitor at a viewing distance of about $57 \mathrm{~cm}$.

Stimuli. Four sets of compound stimuli, black on a white background, were used. Stimuli comprised either a large letter E or a large letter $H$ made up of small circles, as shown in Figure 2. For Stimulus Set A, the small circles were grouped by proximity. These circles were then connected with lines to form Stimulus Set B, in which the circles were grouped by both proximity and UC. Stimulus Sets A and B were embedded in a background composed of small square figures to form Stimulus Sets $C$ and $D$, respectively. The vertical and horizontal sizes of each of the background squares were the same as those of a small circle. The distance between the center of two adjacent circles was equivalent to that between a circle and its neighboring square. The background elements should group by proximity with the target circles making up the global letters; hence, the global letters in Set $C$ were formed by similarity of shapes between constituents. The global letters in Set D, however, were formed by both similarity of shapes and UC. The small circles were arranged in a $6 \times 7$ matrix. The large letter was $4.2 \times 5.4 \mathrm{~cm}$, and the small circle was $0.4 \times 0.4 \mathrm{~cm}$. The large letter and the small circles subtended visual angles, respectively, of $4.2^{\circ} \times 5.4^{\circ}$ and $0.4^{\circ} \times$ $0.4^{\circ}$. The whole pattern with background was $5.8 \times 7.0 \mathrm{~cm}$, sub- tending an angle of $5.8^{\circ} \times 7.0^{\circ}$. The lines forming the small circles and squares and connecting the circles had the same width of 1 pixel.

Each trial began with a 1,000 -msec warning beep and a presentation of the plus-shaped fixation located at the center of the screen. The fixation was $0.2 \times 0.3 \mathrm{~cm}$ subtending $0.23^{\circ} \times 0.3^{\circ}$ of visual angle. After another $1,000 \mathrm{msec}$, the fixation was overlapped by the stimulus display, which was presented at the center of the screen. The stimulus display lasted for $160 \mathrm{msec}$ and was replaced by a square mask formed by random black dots lasting for $80 \mathrm{msec}$. The mask was $7.6 \times 8.2 \mathrm{~cm}$, subtending $7.6^{\circ} \times 8.2^{\circ}$ of visual angle. The time interval between the offset of stimulus display and the onset of mask (interstimulus interval, ISI) was $16,32,80$, or $144 \mathrm{msec}$.

Procedure. The experiment employed a three-factor withinsubjects design, with the factors being proximity (targets were presented with or without background patterns), UC (the circles composing target letters were connected with lines or were not), and ISI. The subjects were required to discriminate the target letters (H vs. E) regardless of how the small circles were grouped. While maintaining their fixation, the subjects were required to respond to the letter $\mathrm{H}$ or the letter $\mathrm{E}$ by pressing one of two keys on a standard keyboard with either the right or the left middle finger. Nine subjects responded to the letter $\mathrm{H}$ with the left middle finger and to the letter $\mathrm{E}$ with the right middle finger. The other subjects responded in the reverse arrangement. After 48 trials for practice, each subject performed four blocks of 144 trials. The subjects were encouraged to respond as quickly and accurately as possible.

RTs and error rates were subjected to a repeated measure analysis of variance (ANOVA) with three factors: proximity, UC, and ISI. Error rates were transformed with an arcsine square-root function before statistical analysis.

\section{Results and Discussion}

Error analysis. The mean error rates for the four sets of stimuli are shown in Figure 3. An ANOVA indicated a main effect of proximity $[F(1,16)=47.07, p<.0005]$, reflecting the fact that the subjects made more errors in responding to the target letters when they were presented with background patterns than when they were presented without background patterns. There was also a main effect of UC $[F(1,16)=69.24, p<.0005]$, indicating that the subjects made fewer errors when the small circles were connected than when they were not connected. There was no significant effect of ISI $(F<1)$ and no reliable two-way interaction involving ISI $(p>.05)$. However, the interaction of proximity and UC reached significance $[F(1,16)=42.84, p<.0005]$; the effect of UC on errors was larger when the small circles were grouped by similarity than when they were grouped by proximity. Finally, the triple interaction between the three factors was also significant $[F(3,48)=3.22, p<.03]$. The difference between the effects of UC on proximity- and similarity-grouped elements was larger at shorter ISIs than at longer ISIs. A further planned orthogonal contrast test showed that the UC effect was significant only with elements grouped by similarity $[F(1,16)=72.75$, $p<.0005]$, but not with elements grouped by proximity $(F<1)$.

RT analysis. The mean RTs for correct responses to the four sets of stimuli are shown in Figure 4. Analysis of the RT data indicated a main effect of proximity $[F(1,16)=$ $122.6, p<.0005]$; responses were faster for target letters 


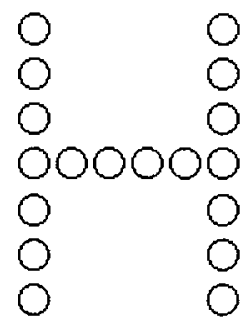

$\operatorname{set} A$

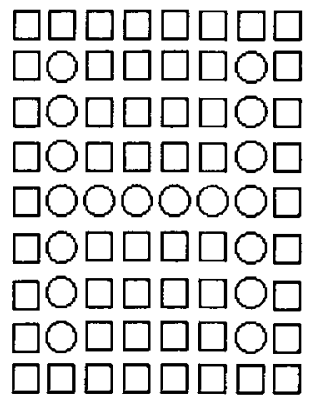

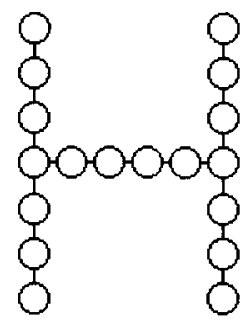

set $B$

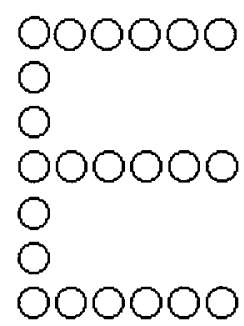

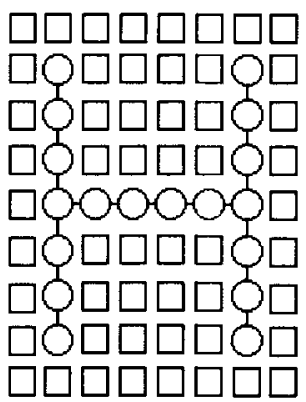

set $D$

Figure 2. Stimuli used in Experiment 1 . The small circles were grouped by proximity (Set $\mathbf{A}$ ), both proximity and UC (Set B), similarity (Set C), and both similarity and UC (Set D).

without background patterns than for targets with background patterns. The main effect of UC was also significant $[F(1,16)=33.16, p<.0005]$, indicating that RTs for targets composed of connected small circles were faster than RTs for targets composed of unconnected small circles. There was no significant effect of ISI $[F(3,48)=2.47$, $p>.07]$, and its interactions with the other two factors were not reliable $(p>.1)$. However, the interaction of proximity and $\mathrm{UC}$ was significant $[F(1,16)=31.69, p<$ $.0005]$. The effect of UC on RTs was larger when the small circles were grouped by similarity than when they were grouped by proximity. The triple interaction of the three factors was not significant $(F<1)$. A further planned orthogonal contrast test showed that the UC effect was significant only under similarity grouping conditions $[F(1,16)=33.56, p<.0005]$, but not when there was grouping by proximity $[F(1,16)=2.81, p>.1]$.

The results primarily showed a difference between the proximity grouping and the similarity grouping conditions (i.e., RTs were much faster when compound letters were formed by proximity grouping than when they were formed by similarity grouping). Error rates were also lower for stimuli grouped by proximity than for those grouped by similarity. These results are in agreement with previous findings (Ben-Av \& Sagi, 1995; Chen, 1986; Han et al., in press), thus providing more evidence for the assertion that proximity grouping occurs earlier or is perceived faster than grouping by similarity of shapes.

The effect of UC grouping on the recognition of target letters was not significant for stimuli formed by proximity grouping. For both RT and error measures, there was no difference between target letters formed by proximity grouping and those formed by both proximity grouping and UC. Grouping by UC affected responses to the stimuli formed by similarity grouping only. RTs for target letters formed by similarity grouping were slower, and error rates were higher, relative to when similarity grouping was augmented by UC to form the stimuli. Interestingly, in comparison with the results from the conditions with proximity grouping alone and with proximity grouping combined with UC, RTs in the condition with similarity and UC grouping were still slower (the average RTs were $569 \mathrm{msec}$ for stimuli with similarity and UC and $488 \mathrm{msec}$ for stimuli grouped by proximity).

Overall, these results indicate that grouping by UC can facilitate grouping by similarity. Nevertheless, there was 

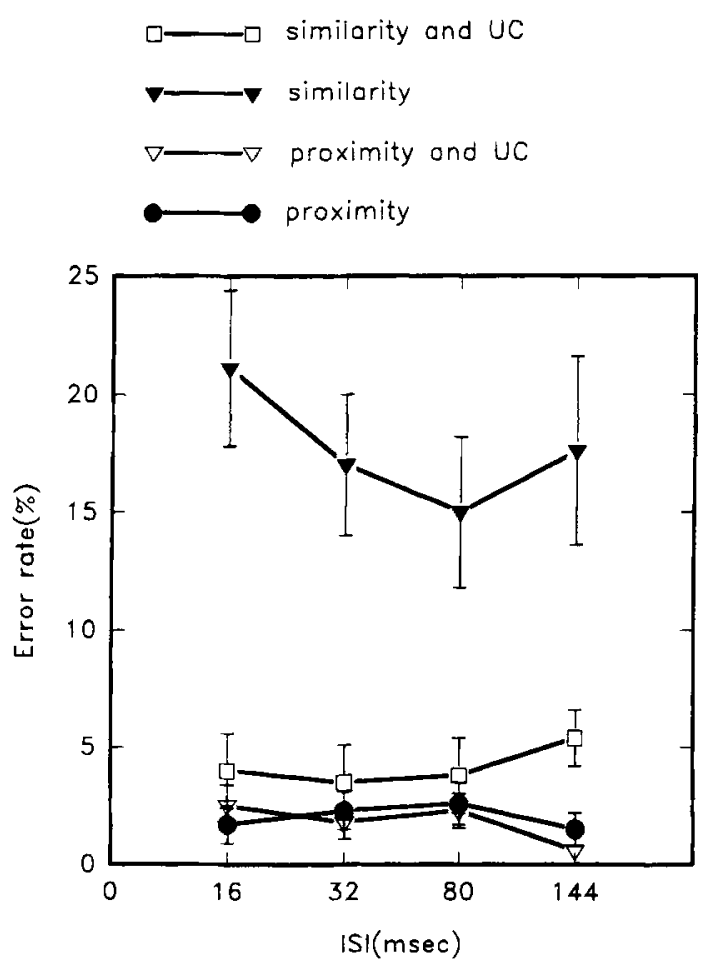

Figure 3. Mean error rates as a function of ISI for each of the four sets of stimuli in Experiment 1.

no evidence for stronger grouping by UC than by proximity. Thus, it appears that proximity grouping is at least as efficient as grouping by UC under the present conditions.

It may be argued that the reason why UC did not facilitate proximity grouping was that the lines connecting the small circles were too faint to be perceived. However, this account can be dismissed because the results in the similarity grouping condition provide evidence that the connecting lines were perceived well enough to augment similarity grouping. It may also be possible that the lines connecting local circles were slow to be perceived and so had an effect only with slow similarity grouping, but not with fast proximity grouping. However, this cannot be true because the data in a supplementary experiment (see the Appendix) showed that target letters composed only of the lines were responded to as quickly as target letters in the proximity grouping condition here. Therefore, the similar RTs to Stimulus Sets A and B in the present experiment were due not to the connecting lines' being relatively difficult to discriminate but to connectedness being no more efficient than proximity in grouping the local circles into global targets.

\section{EXPERIMENT 2}

The topological approach to visual perception (Chen, 1982) claims that the perception of topological properties of displays (e.g., closure) occurs earlier than that of local geometrical properties (e.g., orientation coding). Conse- quently, similarity grouping based on topological properties should take place prior to similarity grouping based on the local geometrical properties of elements. The circles making up the letters and the squares making up the background in Experiment 1 were both closed patterns, and this may have prevented grouping based on closure from occurring. The purpose of Experiment 2 was to examine further the difference between grouping based on proximity, $\mathrm{UC}$, and similarity, but this time testing similarity when closure may be used. The stimuli from Set A in Experiment 1 were embedded in a background made up of plus elements. The circles, being closed patterns, differ topologically from the background pluses and may be grouped by similarity of closure. We compared RTs to discriminate target letters composed of small circles that were grouped together on the basis of proximity, similarity of closure, and both UC and similarity of closure.

\section{Method}

Subjects. The subjects were 3 female and 12 male undergraduate and graduate students ( $20-24$ years of age) from the Graduate School of University of Science and Technology of China, who were paid volunteers. All subjects were right-handed and had normal or corrected-to-normal vision.

Apparatus, Stimuli, and Procedure. All aspects were the same as those in Experiment 1 except that there were three sets of stimuli in Experiment 2, illustrated in Figure 5. Stimulus Set A was the same as Set A in Experiment 1. Stimulus Set B was formed by embedding Set $\mathrm{A}$ in a background composed of small plus figures. Each of the pluses had the same vertical and horizontal size as the circle. Stimulus Set $\mathrm{C}$ was formed by connecting all the small cir-

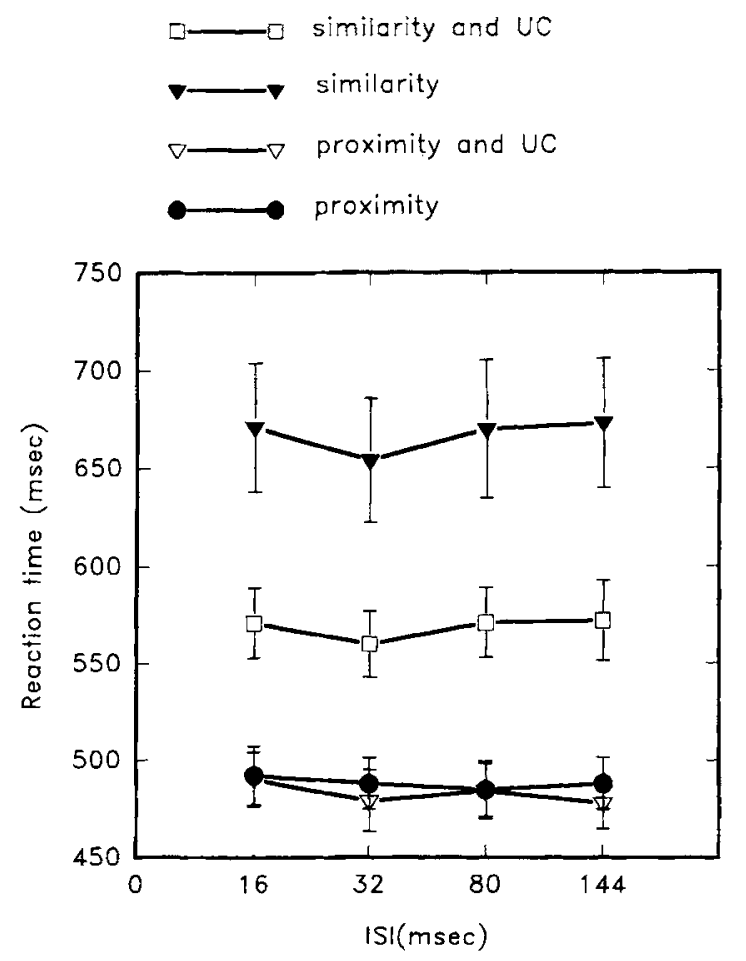

Figure 4. Mean reaction times as a function of ISI for each of the four sets of stimuli in Experiment 1. 

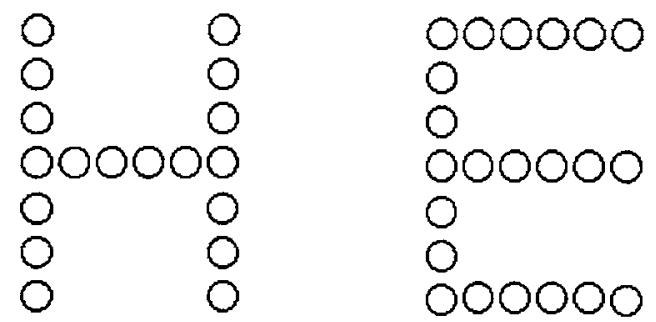

set $A$

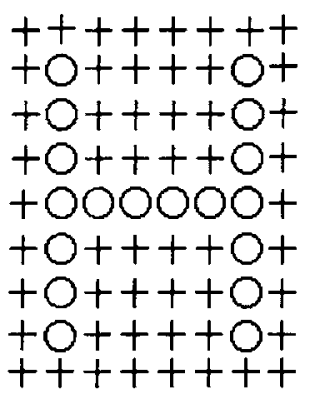

$+++++++$ to00000t $+\mathrm{O}+\mathrm{t}+\mathrm{t}+$ $+\mathrm{O}+\mathrm{t}+\mathrm{t+}$ +OOOOOO+ $+\mathrm{O}+\mathrm{t+}+\mathrm{+}$ $+\mathrm{O}+\mathrm{t}+\mathrm{t+}$ +000000+ $++++++t$ set $B$
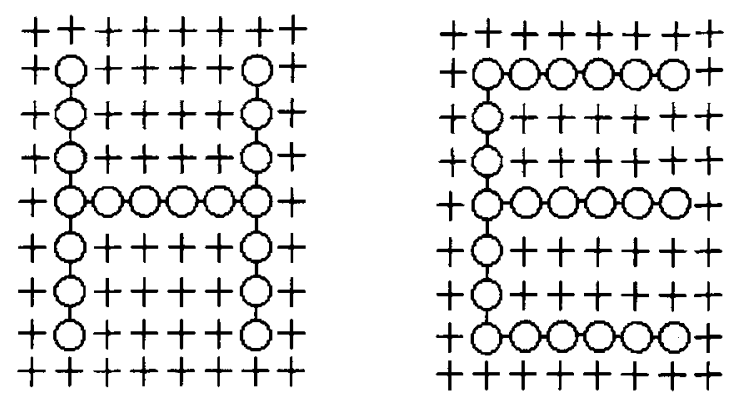

set A

Figure 5. Stimuli used in Experiment 2. The small circles were grouped by proximity (Set A), similarity of closure (Set B), and both similarity of closure and UC (Set $C$ ).

cles from the stimuli in Set $B$. There were 48 practice trials and 288 experimental trials divided into three blocks of 96 trials each.

\section{Results and Discussion}

Error analysis. Figure 6 shows the mean error rates for the three sets of stimuli. There was a significant main effect of grouping $[F(2,28)=7.06, p<.003]$. The effect of ISI $[F(3,42)=1.37, p>.25]$ and the interaction of these two factors did not reach significance $[F(6,84)=$ $1.39, p>.2$ ]. Further univariate $F$ tests showed that error rates to Stimulus Set A (grouped by proximity) were lower than those to Stimulus Sets B and C $[F(1,14)=11.52, p<$ $.004]$. There was no significant difference between error rates to Stimulus Sets B and C $[F(1,14)=3.69, p>.07]$.
RT analysis. Average RTs for correct responses to the three sets of stimuli are shown in Figure 7. There were main effects of grouping $[F(2,28)=140.40, p<.0005]$ and ISI $[F(3,42)=3.80, p<.02]$. The interaction between the two factors was also significant $[F(6,84)=4.29, p<$ $.001]$. Further univariate $F$ tests showed that RTs to Stimulus Set A (grouped by proximity) were faster than those to Stimulus Sets B and C $[F(1,14)=172.23, p<.0005]$. RTs to Stimulus Set B (grouped by similarity of closure) were slower than RTs to Stimulus Set C (grouped by both $\mathrm{UC}$ and similarity of closure) $[F(1,14)=86.29, p<.0005]$.

The results of Experiment 2 showed that RTs to stimuli formed by proximity grouping were faster than those to stimuli formed by grouping by similarity and UC. RTs in the latter condition were faster than those for the stimuli grouped by similarity of closure alone. The interaction with ISI occurred because the advantage for stimuli grouped by proximity was particularly large at the short ISI. Hence, there is evidence for faster grouping by proximity than by both UC and similarity of closure. Nevertheless, UC between elements did facilitate performance relative to the condition in which elements were grouped by similarity of closure alone. Therefore, UC dominates grouping by similarity even when similarity is defined by a topological property.

There was also a suggestion that grouping by similarity operates over a longer time course than grouping by proximity or UC, since errors showed a larger decrease over time in the similarity condition (though the inter-
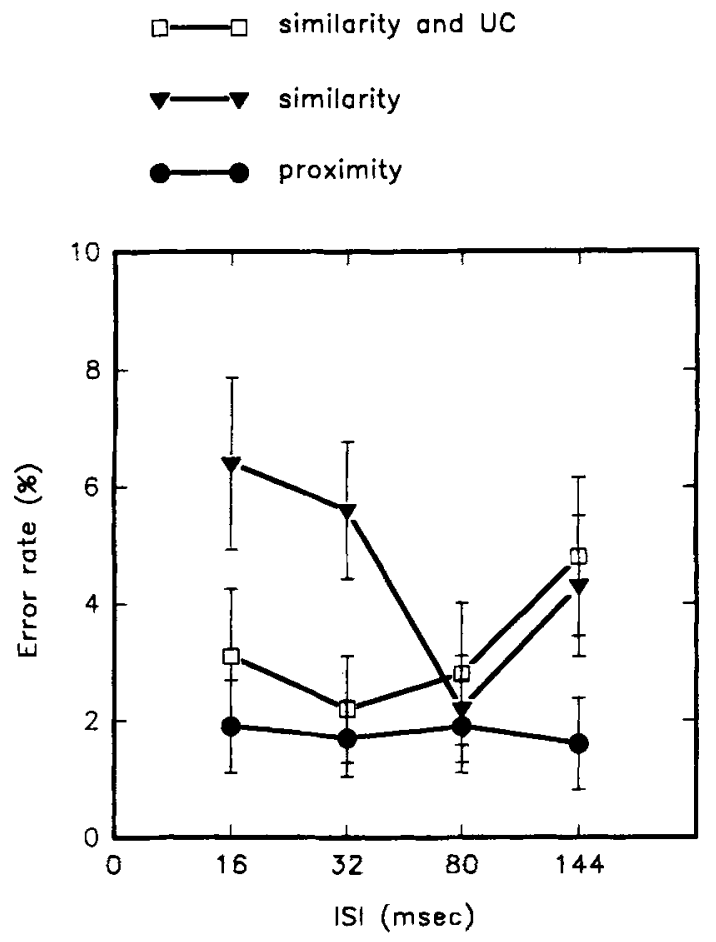

Figure 6. Mean error rates as a function of ISI for each of the three sets of stimuli in Experiment 2. 


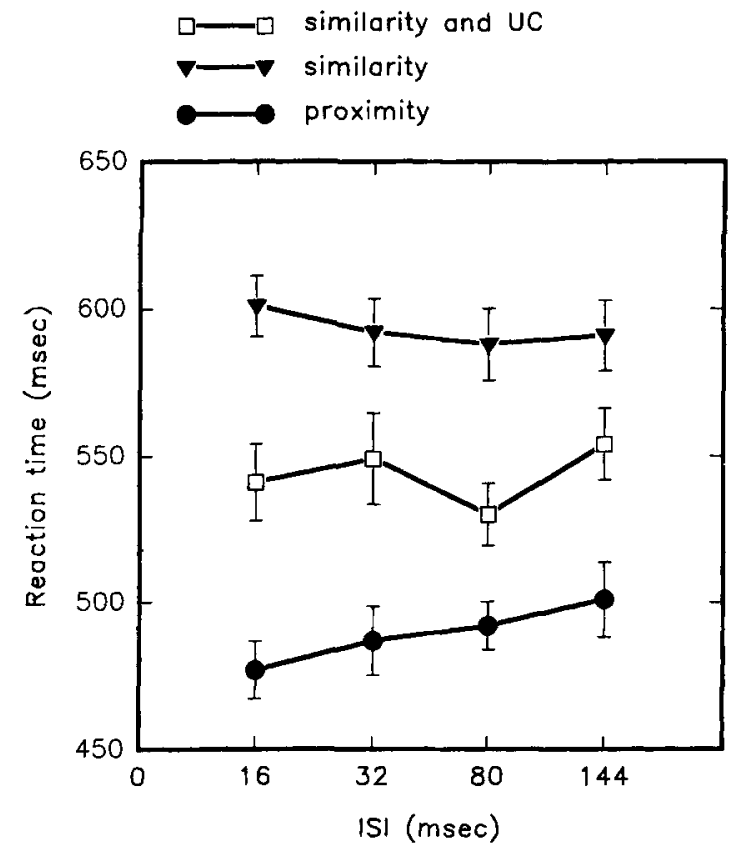

Figure 7. Mean reaction times as a function of ISI for each of the three sets of stimuli in Experiment 2.

action between grouping and ISI was not reliable). Also, relative to Experiment 1 in which error rates were reduced (particularly for the similarity grouping condition), error rates in the similarity grouping condition were not affected by UC in Experiment 2 . This suggests that the perceptual quality of the target letters was the same for stimuli grouped by proximity, similarity of closure, and both $\mathrm{UC}$ and similarity of closure. UC can facilitate grouping by similarity of topological and local geometrical properties alike, but its effect on the perceptual quality of target letters is greater for stimuli grouped by local geometrical similarity (as in Experiment 1) than for stimuli grouped by closure (as here).

\section{EXPERIMENT 3}

The purpose of Experiment 3 was to examine possible alternative interpretations for the results of Experiments 1 and 2. For instance, in the earlier conditions with proximity grouping, the target letters were presented on a blank background, whereas, in the similarity grouping conditions, they were presented on a background composed of squares or crosses. It may be argued that the slow responses in the latter conditions were due to the fact the similarity conditions involved an extra processing stage: figure-ground segmentation. ${ }^{1}$ To rule out this factor, Experiment 3 used similarity and proximity conditions in which the stimulus patterns were exactly the same, except that the distances between local elements were manipulated. When local elements are evenly spaced, grouping by similarity may dominate; however, when some elements are spaced closer than others, proximity grouping may overrule similarity grouping (e.g., see Bruce, Green,
\& Georgeson, 1996). In addition, since proximity is a continuous variable, Experiment 3 investigated whether UC can dominate proximity when the latter is not strong enough, by manipulating the strength of proximity grouping. Finally, a longer ISI than those in Experiments 1 and 2 was used to study the effect of ISI further, because it may be possible that the ISIs were too short to show their effects in Experiments 1 and 2.

\section{Method}

Subjects. The subjects were 20 male undergraduate and graduate students (20-24 years of age) from the Graduate School of University of Science and Technology of China, who were paid volunteers. All subjects were right-handed and had normal or corrected-to-normal vision.

Apparatus. Data collection and stimulus presentation were controlled by a 586 personal computer. Stimuli were presented on a $12-$ in. color monitor.

Stimuli. Four sets of stimuli, shown as black on a white background, were used. Stimuli consisted of square arrays of small filled circles and squares arranged in a $8 \times 8$ matrix, as illustrated in Figure 8 . Each circle or square was $0.4 \times 0.4 \mathrm{~cm}$. The whole stimulus pattern was $11.0 \times 11.0 \mathrm{~cm}$. At a viewing distance of about $70 \mathrm{~cm}$, the whole stimulus pattern and the unit shape subtended visual angles, respectively, of $8.99^{\circ} \times 8.99^{\circ}$ and $0.33^{\circ} \times$ $0.33^{\circ}$. There was only one type of shape (circle or square) in each column or row in the stimulus patterns.

For Stimulus Set A, the distance between two adjacent columns was equivalent to that between two adjacent rows; thus, the circles or squares were grouped into columns or rows based on similarity. The size of the whole array was kept constant, and the distances between the two adjacent columns or rows composed of the same shapes as in Stimulus Set A were adjusted in the following way to form Stimulus Sets B and C: When the circles or squares were in rows (or columns), two adjacent rows (or columns) were moved close to each other so that four horizontal (or vertical) groups were formed on the basis of the proximity of two adjacent rows (or columns). The horizontal or vertical groups based on proximity were always congruent with the groups formed by similarity. For Stimulus Set B, the spacing ratio between two near rows (or columns) and two far rows (or columns) was .5. For Stimulus Set C, this spacing ratio was .1. Therefore, proximity grouping in Stimulus Set $C$ was stronger than in Stimulus Set B. Stimulus Set D was made by connecting the circles (or squares) in the same column (or row) as in Stimulus Set A with lines. Each of the lines was 1 pixel in width. Hence, the circles and squares were grouped together on the basis of both similarity of shapes and UC. In summary, the horizontal or vertical groups were formed by similarity of shapes for Stimulus Set A, by weak proximity plus similarity for Stimulus Set B, by strong proximity plus similarity for Stimulus Set C, and by UC plus similarity for Stimulus Set $D$.

Procedure. Each trial began with a $250-\mathrm{msec}$ warning beep and a presentation of the plus-shaped fixation located at the center of the screen. The fixation cross was $0.2 \times 0.3 \mathrm{~cm}$ subtending $0.23^{\circ}$ $\times 0.3^{\circ}$ of visual angle. After another $500 \mathrm{msec}$, the fixation cross was overlapped by the stimulus display presented at the center of the screen. The stimulus display lasted for $160 \mathrm{msec}$ and was replaced by a square mask formed by random black dots lasting for $80 \mathrm{msec}$. The mask was $13.5 \times 13.5 \mathrm{~cm}$, subtending $11.0^{\circ} \times 11.0^{\circ}$ of visual angle. The ISI was either 32 or $304 \mathrm{msec}$.

The subjects were required to discriminate how the local shapes (circles or squares) were arranged (either in columns or in rows), regardless of how they were grouped. While maintaining their fixation, the subjects were instructed to respond to the horizontal or vertical groups by pressing one of the two keys on a standard keyboard with the right or the left middle finger. The relationship between the stimuli and responding hand was counterbalanced across subjects. 


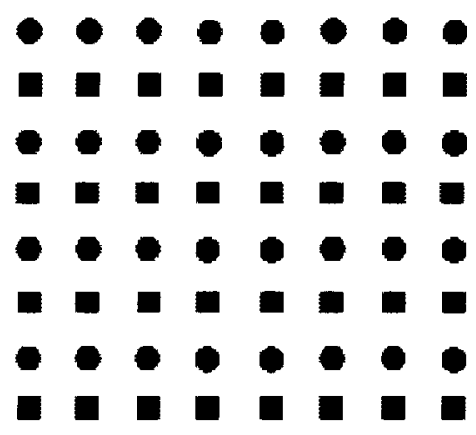

set A

\section{i: : : : : : \\ $9: 1,1: 1$ \\ $191,1: 1:$ \\ : : : : : : : \\ set C}

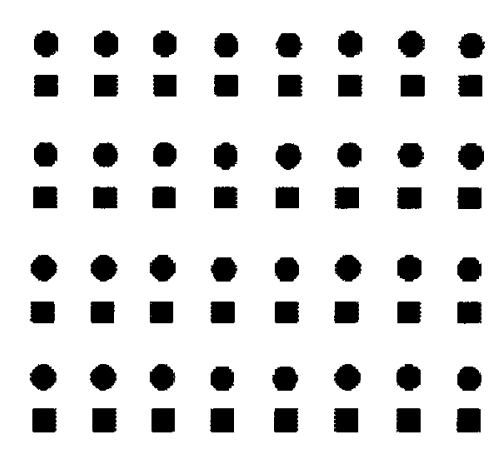

set B

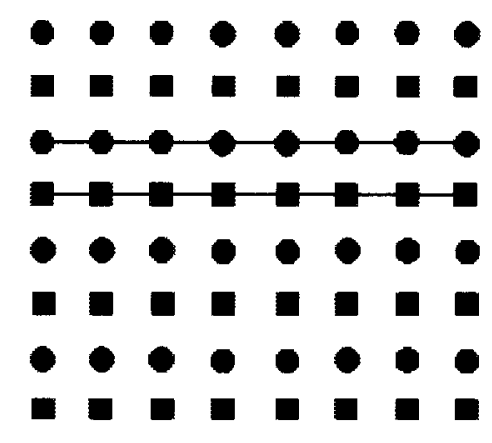

set $\mathrm{D}$

Figure 8. Illustration of half of the stimuli used in Experiment 3. The horizontal groups were formed respectively by similarity of shapes (Set $A$ ), similarity plus weak proximity (Set B), similarity plus strong proximity (Set C), and similarity plus UC (Set D). The circles and squares were grouped vertically for the other half of the stimuli.

After 32 trials for practice, each subject performed four blocks of 80 trials. The subjects were encouraged to respond as quickly and accurately as possible.

To ensure that the subjects could perceive the connecting lines clearly, each subject was asked to report the orientation of the lines (horizontal or vertical) in a block of 16 trials at the end of the experiment. No subjects made any errors in this task. Similarly, no errors were made by the subjects viewing the four types of display under unlimited viewing times; under these conditions, the formation of the horizontal and vertical groups was unambiguous.

RTs and error rates were subjected to a repeated measure ANOVA with two factors: grouping (horizontal or vertical groups were formed by similarity of shapes, weak proximity plus similarity, strong proximity plus similarity, or UC plus similarity) and ISI. Error rates were transformed with an arcsine square-root function before statistical analysis.

\section{Results and Discussion}

Error analysis. The mean error rates for the four sets of stimuli are shown in Figure 9. ANOVA indicated only a main effect of grouping $[F(3,57)=6.10, p<.001]$, reflecting the fact that the error rates were higher for Stimulus Set A (similarity grouping) than for the others. The effect of ISI and its interaction with grouping were not significant $(p>.05)$.

RT analysis. The mean RTs for correct responses to the four sets of stimuli are shown in Figure 10. An ANOVA on the RT data indicated a main effect of grouping $[F(3,57)=31.16, p<.0005]$. The effect of ISI was also significant $[F(1,19)=33.55, p<.0005]$, indicating that RTs in the short-ISI conditions were faster than those in the long-ISI conditions. The interactions between grouping and ISI were also significant $[F(3,57)=16.26, p<$ $.0005]$, reflecting the fact that the effect of ISI was different for the four stimulus sets.

Further separate comparisons showed that RTs for Stimulus Set A (similarity grouping) were slower than those for Stimulus Set B (weak proximity + similarity) $[F(1,19)=52.58, p<.0005]$. RTs for Stimulus Set B were, in turn, slower than those for Stimulus Set C (strong proximity + similarity) $[F(1,19)=18.96, p<.0005]$ and Stimulus Set D (UC + similarity) $[F(1,19)=6.351, p<$ $.02]$. There was no significant overall difference between RTs for Stimulus Sets C and D $(F<1)$; however, the ef- 


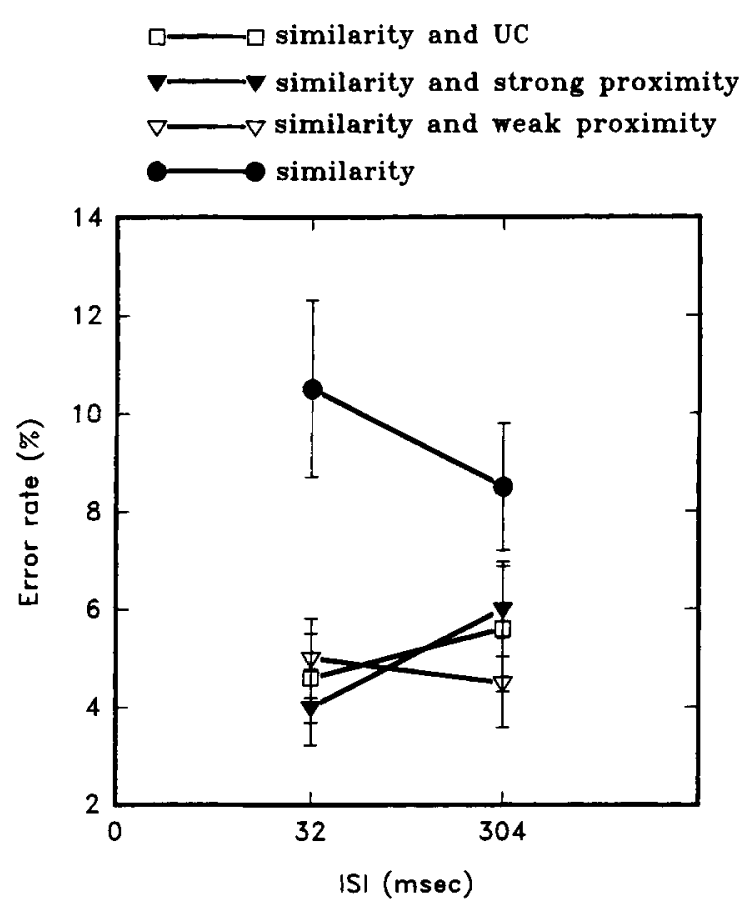

Figure 9. Mean error rates as a function of ISI for each of the four sets of stimuli in Experiment 3.

fect of ISI was stronger for Stimulus Set D (UC + similarity) than for Stimulus Set C (strong proximity + similarity) $[F(1,19)=8.376, p<.009]$ and for Stimulus Set B (weak proximity + similarity) $[F(1,19)=14.52, p<.001]$. The smallest effect of ISI was on Stimulus Set A (similarity grouping) $[F(1,19)=9.539, p<.006]$ (for comparison with Stimulus Set B).

In Experiment 3, local elements were not embedded in background stimuli; consequently, the process of figureground segmentation was unlikely to have been as critical as it might have been in the earlier studies. Nevertheless, error rates were higher and RTs were longer to stimuli defined by shape similarity (Set A) than to those defined by either proximity (Sets B and C) or connectedness (Set D). Stimuli defined by connectedness were responded to faster than those defined by weak proximity, but not faster than those defined by strong proximity. There was also some evidence again for the time course of grouping being different for the different forms of grouping. Generally, RTs slowed as the ISI increased, but the ISI effect was smallest on the similarity-grouping condition. This may have been because, for this condition, the subjects always made responses on the basis of the information produced by similarity grouping irrespective of how long the ISIs were. For the other three conditions, however, when the ISI was short, responses appeared to be based on the information produced by proximity or UC grouping. When there was a long ISI, however, the subjects may have delayed responding in order to have the maximal amount of infor- mation available, including that produced by similarity grouping (derived over a longer time course).

The data again indicate that similarity of shape is less efficient in perceptual grouping than either proximity or UC. Moreover, the RT results suggest that proximity was not always as strong as UC for grouping, though this is a matter of degree. Proximity grouping can be as fast and efficient as grouping by UC when elements are close enough.

One question concerns why responses in the similarity and UC grouping conditions in Experiments 1 and 2 were slower than those in the proximity grouping conditions. A probable account is that the local circles comprising the target letters in Experiments 1 and 2 were grouped together with the surrounding squares (or pluses) by proximity, even when they were connected with lines. The grouping between the circles and squares (or pluses) by strong proximity competed with the grouping between the circles by $\mathrm{UC}$ and thus weakened the effect of UC groups on RTs. Such competitive grouping effects were removed here by omitting the background elements.

\section{EXPERIMENT 4}

Palmer and Rock (1994) claimed that the main difference between UC and classical grouping principles is that UC does not require any "putting together" of separate elements because there are no such separate elements prior to its application. Hence, grouping by UC may be more efficient than other forms of grouping. We have shown that

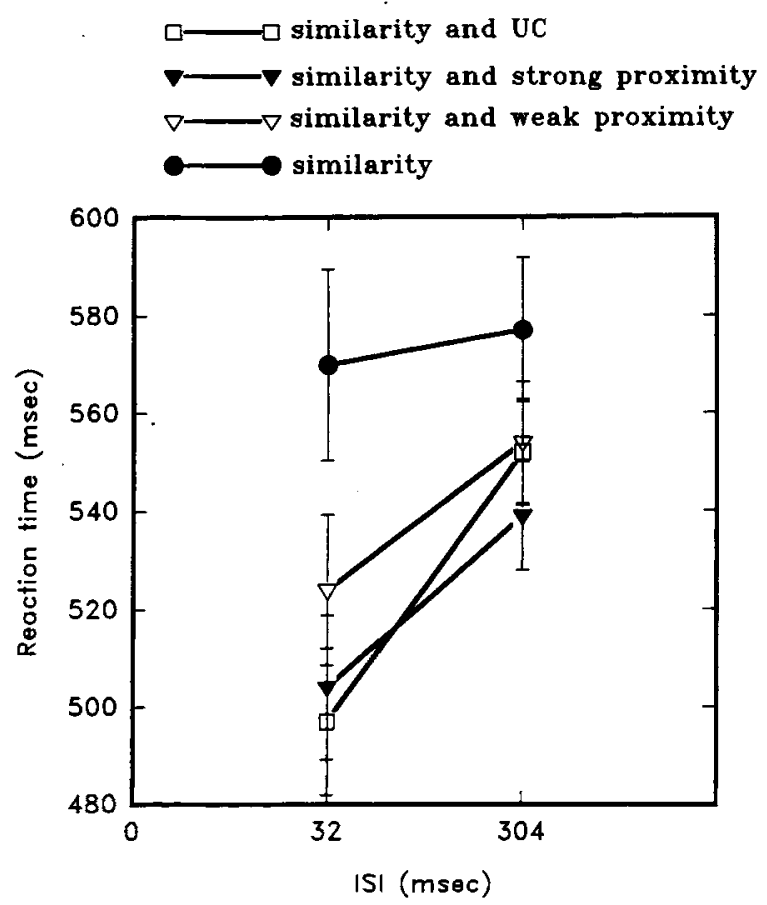

Figure 10. Mean reaction times as a function of ISI for each of the four sets of stimuli in Experiment 3. 

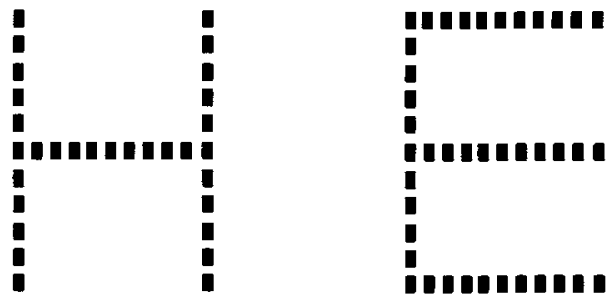

set A
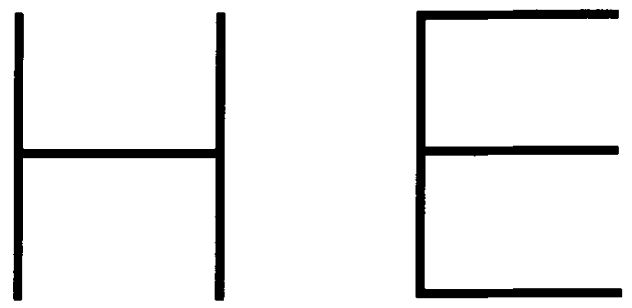

set $B$

Figure 11. Stimuli used in Experiment 4.

grouping by UC can be more efficient than grouping by similarity and weak proximity but that it is not necessarily more efficient than grouping by strong proximity. However, it might be that the lines connecting local elements in Experiments 1-3 were not wide enough to make UC distinguishable from strong proximity grouping, though the subjects could discriminate the lines clearly. The ultimate way to test the above possibility is to use stimuli composed of solid lines. Are stimuli composed of solid lines grouped and discriminated faster than those determined by strong proximity grouping, even when the gaps between the grouped elements can be easily discriminated? In Experiment 4, we tested letter discrimination with elements grouped by proximity relative to a solid-line baseline condition to give the best chance for precedence of grouping by UC over that by proximity. We designed two sets of large letters ( $\mathrm{H}$ and $\mathrm{E})$ made up of solid lines or separated small solid rectangles, and had subjects participate in a letter identification task. In order to rule out the possibility that subjects could not perceive the gaps between two adjacent rectangles when the stimuli were briefly presented and masked, a control condition was also administered in which subjects had to discriminate whether the target letters were made up of solid rectangles or were merely solid lines.

\section{Method}

Subjects. The subjects were 8 female and 8 male undergraduate psychology students (19-27 years of age) from the University of Birmingham, who were paid volunteers. Three subjects were lefthanded; the others were right-handed. All had normal or correctedto-normal vision. Fourteen of the subjects took part in the control task.
Apparatus, Stimuli, and Procedure. The apparatus and procedure were the same as used in Experiment 1. Two sets of stimuli, black on a white background, were used, making up either a large letter E or a large letter H (shown in Figure 11). For Stimulus Set A, the letters were made up of small solid rectangles, which were grouped together on the basis of their proximity. The small rectangles were arranged in an $11 \times 11$ matrix. The large letter was $4.2 \times 5.4 \mathrm{~cm}$, and each small rectangle was $0.25 \times 0.40 \mathrm{~cm}$. The large letter and the small rectangles subtended visual angles, respectively, of $4.2^{\circ} \times$ $5.4^{\circ}$ and $0.25^{\circ} \times 0.40^{\circ}$. For Stimulus Set $B$, the letters were made up of solid lines, as shown in Figure 11. The thicknesses of the vertical and horizontal lines composing Stimulus Set B were 2.0 and $2.5 \mathrm{~mm}$, respectively. The size of each of the large letters in Set B was the same as that of the letters in Set A. The total areas of letters $H$ and $E$ when made up of solid rectangles were 310 and $380 \mathrm{~mm}^{2}$, respectively. The total areas of letters $\mathrm{H}$ and $\mathrm{E}$, when composed of solid lines, were 306 and $378 \mathrm{~mm}^{2}$, respectively. The subjects were asked to discriminate whether the target letter was $\mathrm{H}$ or $\mathrm{E}$. For the control condition, the subjects were asked to discriminate whether the target letters were made up of solid rectangles or solid lines, but there was only one ISI condition ( $16 \mathrm{msec})$.

After a practice set of 16 trials, each subject was given two blocks of 96 trials. Eight subjects responded to the target letter $\mathrm{H}$ with the left hand and to the target lctter $\mathrm{E}$ with the right hand; the other subjects were given the reverse arrangement. For the control condition, each subject was given one block of 60 trials. The first 12 trials were for practice. Half of the subjects responded to target letters made up of solid rectangles with the left hand and to target letters composed of solid line stimuli with the right hand; the other subjects were given the reverse arrangement.

\section{Results and Discussion}

Error analysis. The mean error rates are shown in Figure 12. There was a reliable main effect of ISI $[F(3,45)=$ $2.89, p<.05]$. The effect of grouping and its interaction with ISI were not significant $(F<1)$.

RT analysis. The mean RTs for correct responses to the two sets of stimuli are given in Figure 13. The effects of grouping $[F(1,15)=2.05, p>.15]$ and $\operatorname{ISI}[F(3,45)=$ $2.20, p>.1]$ were not significant, nor was the grouping $\times$ ISI interaction $[F(3,45)=1.12, p>.3]$.

RTs and error rates in the letter identification condition with the shortest ISI were compared with those in the control discrimination condition (is the stimulus connected or not?) (see Table 1). Paired $t$ tests indicated no difference between RTs and error rates in the two conditions. Importantly, error rates were very low in the control condition; the subjects could discriminate whether single lines or separated elements were presented.

The present experiment again did not show any difference between stimuli formed by UC and by proximity, contrary to Palmer and Rock's (1994) assertion that UC operates prior to proximity in perceptual grouping. There was a small trend for a benefit for UC stimuli at the shortest ISIs, but this did not approach significance. Overall, it appears that proximity is as efficient as UC in perceptual grouping here.

\section{GENERAL DISCUSSION}

The results of the present study show that the recognition of target letters formed by proximity grouping is more efficient than that of target letters formed by group- 


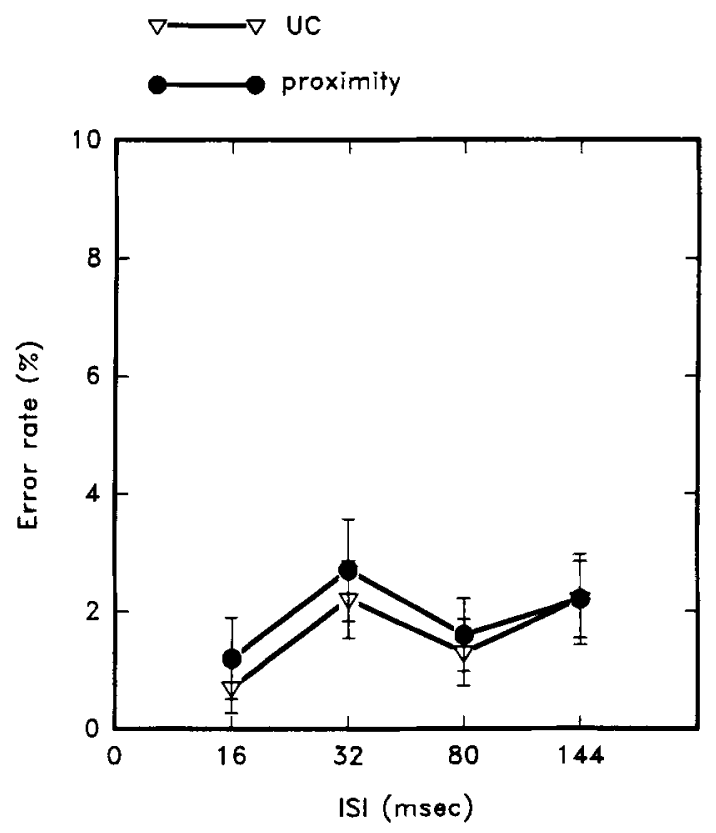

Figure 12. Mean error rates as a function of ISI for each of the two sets of stimuli in Experiment 4.

ing by similarity of shapes. The discrimination of the orientations of perceptual groups formed by proximity was faster and more accurate than that of groups formed by shape similarity. The difference between responses to stimuli formed by proximity grouping and by similarity grouping was observed across all ISIs and under conditions in which the difficulties of figure-ground segmentation were comparable. These results are consistent with the notion that proximity dominates similarity of shapes at early stages of perceptual grouping (Ben-Av \& Sagi, 1995; Chen, 1986; Han et al., in press).

The present data also show that grouping by UC (1) speeded RTs and decreased error rates for stimuli grouped by similarity of local geometrical properties (Experiments 1 and 3 ) and (2) speeded RTs (without affecting accuracy) for stimuli grouped by similarity of closure (Experiment 2). These results are in agreement with Palmer and Rock's (1994) hypothesis that grouping by UC can overcome grouping by similarity either by generating stronger groups or by occurring earlier. However, our data indicate that grouping by proximity can be as efficient as grouping by UC. RTs to global letters defined by proximity grouping alone were as fast as RTs to global letters defined by proximity and UC (Experiment 1) and even to stimuli that were fully connected straight lines (Experiment 4). In Experiment 3, responses to discriminate the orientations of perceptual groups formed by strong proximity (Stimulus Set C) and UC (Stimulus Set D) were equally rapid, though UC grouping was shown to be faster than grouping by weak proximity (Stimulus Set B). Therefore, the results lend no support to the assertion that group- ing starts from UC regions and that grouping by proximity always occurs at a later stage.

One hypothesis to account for the domination of proximity over similarity in grouping is that proximity grouping does not require that the features of visual elements (their shape, size, etc.) be clearly registered; rather, the constituent elements may function just as "place holders" (Treisman, 1986). The recognition of a global form, needed for the present experiments, depends only on the representation of the spatial relationships between the local elements. For similarity grouping, however, some features of the local elements, or feature differences between elements composing the target letters and the background patterns, must be represented before grouping operates. Differences in the speed and efficiency of grouping by similarity and proximity may be determined by the time needed to compute the critical visual features.

The above hypothesis is in line with Pomerantz's (1981, 1983) distinction between two types of visual configuration. Pomerantz proposed that, in Type $\mathrm{P}$ configurations, only the position of the local elements matters for the identity of the global patterns. In Type $\mathrm{N}$ configurations, however, not only the position but also the nature of the local elements is important for the identity of the global patterns. In previous studies (Han \& Humphreys, 1997; Miller, 1981), with global shapes formed by proximity grouping, the identities of the local elements have been found not to affect identification of the global forms: Identification times are the same for global letters composed of identical or different local letters. This implies that similarity cannot facilitate RT if grouping is dominated by proximity. In contrast, distorting a global letter by moving a local element away from its original loca-

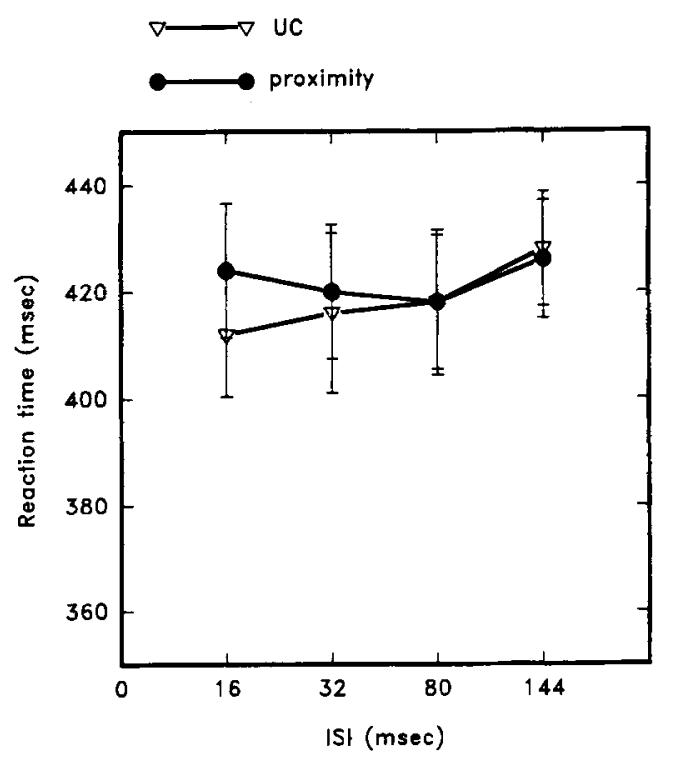

Figure 13. Mean reaction times as a function of ISI for each of the two sets of stimuli in Experiment 4. 
Table 1

Reaction Times (RTs, in Milliseconds) and Error Rates in the Condition With the Shortest Interstimulus Interval (ISI) and in the Control Condition

\begin{tabular}{lcccc}
\hline & $\begin{array}{c}\text { Condition With } \\
\text { Shortest ISI }\end{array}$ & $\begin{array}{c}\text { Control } \\
\text { Condition }\end{array}$ & $t$ & $p>$ \\
\hline RT $(\mathrm{msec})$ & 428 & 427 & 0.03 & .9 \\
Error $(\%)$ & 0.98 & 2.6 & 1.83 & .08 \\
\hline
\end{tabular}

tion decreases global RTs severely (Hoffman, 1980). Thus, global stimuli formed by proximity grouping may depend on Type $P$ information. However, for stimuli formed by similarity grouping, as shown in our study (Han et al., in press), both the location and nature of local elements are critical to the global figure's identity, and the need to compute featural information may delay responses to stimuli based on the grouping of this information. Furthermore, the results in Experiment 3 indicate that proximity (Type $P$ information) can facilitate responses even when similarity is still present (in the conditions with proximity + similarity, relative to similarity alone).

Our finding that grouping by proximity can be as fast as grouping by $\mathrm{UC}$ may be explained in several ways, which we discuss here. One possible reason is that the recognition of global shapes formed both by proximity grouping and by UC is underpinned by low-spatialfrequency channels. Ginsburg $(1973,1986)$ has shown that filtering letters composed of dots or lines by passing only the low spatial frequencies keeps the underlying shape of both types of form. If low-spatial-frequency channels underlie both types of grouping, recognition efficiency should not differ as a function of the type of grouping involved.

A rather different view is that, for both types of grouping, global forms are computed from more local elements that cooperate to form edge boundaries. For instance, Grossberg and Mingolla (1985) have proposed that oriented edge detectors cooperate to form virtual boundaries around shapes, and they will do this even when there are gaps between edges (provided of course that the gaps are sufficiently small and do not span the whole receptive field for a cell). This boundary completion process should operate with fully connected and close elements, minimizing differences between grouping by proximity and by UC. Note, however, that, for this proposed boundary completion system to work maximally, the proximal elements should have aligned edges; when such edges are absent, grouping by boundary completion can break down (see Gilchrist, Humphreys, Riddoch, \& Neumann, 1997, for evidence). While this was the case in the present Experiment 4 (in which we used aligned squares), the circles used in Experiment 1 may nevertheless have been fine enough to enable boundary completion to operate in cells with aligned, oriented receptive fields.

The third possible account of the equality of grouping by proximity and $U C$ is that both types of grouping reflect a basic operation by our perceptual system - the encoding of connectivity within a perceptual tolerance space (Chen, 1984; Zeeman, 1962). A perceptual tolerance space may be thought of as analogous to a "basin of attraction" within a connectionist network (cf. Hopfield, 1982; see Hinton \& Shallice, 1991, for an application to cognitive psychology). For instance, two unconnected stimuli may fall at nearby locations in the tolerance space for coding connectivity. If they fall within the same basin of attraction, the stimuli will be pushed to its center and henceforth be treated as perceptually equivalent by subsequent recognition processes. If they fall into different basins of attraction (e.g., if they are too far apart), they will be treated as separate elements by the recognition system. Nevertheless, even when stimuli fall in the same basin of attraction, subjects may be able to discriminate them by gaining access to the perceptual information after it is encoded within the tolerance space. Hence, even though identification times for the fully connected and unconnected stimuli were the same in Experiment 4, the subjects were still able to discriminate them.

The three possible mechanisms underlying grouping by proximity and UC are couched at different levels of theoretical interpretation (cf. Marr, 1982). The first two are relatively low level accounts that may deal with how grouping by proximity and UC are realized physiologically. The third account is relatively high level and elucidates how the connectivity of stimuli formed by proximity grouping and UC operates at a computational level. Further studies are needed to clarify whether only one of or all these mechanisms function in perceiving connectivity for stimuli formed by proximity grouping and UC.

\section{REFERENCES}

Ben-Av, M. B., \& SAGI, D. (1995), Perceptual grouping by similarity and proximity: Experimental results can be predicted by intensity autocorrelations. Vision Research, 35, 853-866.

Bruce, V., Green, P., \& GeORGeson, M. A. (1996). Visual perception (3rd ed.). London: Psychology Press.

Chen, L. (1982). Topological structure in visual perception. Science, 218, 699-700.

CHEN, L. (1984). Gestalt laws of proximity and similarity and tolerance space. Acta Psychologica Sinica, 3, 259-266. (In Chinese)

ChEN, L. (1986). Topological perception-A possible dark cloud over computational theory. In Q. X. Sen (Ed.), Essays on cognitive sciences (pp. 250-301). Shanghai: People's Press of Shanghai. (In Chinese)

DUNCAN, J. (1984). Selective attention and the organization of visual information. Journal of Experimental Psychology: General, 113, 501507.

DunCAN. J., \& Humphreys, G. W. (1989). Visual search and stimulus similarity. Psychological Review, 96, 433-458.

Gilchrist, I. D., Humphreys, G. W., Riddoch, M. J., \& NeumanN, H. (1997). Luminance and edge information in grouping: A study using visual search. Journal of Experimental Psychology: Human Perception \& Performance, 23, 18-35.

GinsBurg, A. P. (1973). Pattern recognition techniques suggested from psychological correlates of a model of the human visual system. [Proceedings of the National Aerospace Electronics Conference (NAECON)]. IEEE Transactions on Aerospace \& Electronic System, 73-CH0735, 309-316.

GINSBURG, A. P. (1986). Spatial filtering and visual perception. In K. R. Boff, L. Kaufman, \& J. P. Thomas (Eds.), Handbook of perception and human performance (chap. 34). New York: Wiley.

Grossberg, S., \& Mingolla, E. (1985). Neural dynamics of form per- 
ception: Boundary completion, illusory figures, and neon color spreading. Psychological Review, 92, 173-211.

HAN, S., \& HuMPhrEYs, G. W. (1997). [Effect of grouping by similarity on global precedence]. Unpublished raw data.

HaN, S., Humphreys, G. W., \& Chen, L. (in press). Parallel and competitive processes in hierarchical analysis: Perceptual grouping and encoding of closure. Journal of Experimental Psychology: Human Perception \& Performance.

Hinton, G. E., \& Shallice, T. (1991). Lesioning and attractor network: Investigations of acquired dyslexia. Psychological Review, 98, 74-95.

HoFFMAN, J. E. (1980). Interaction between global and local levels of a form. Journal of Experimental Psychology: Human Perception \& Performance, 6, 222-234.

HopFIELD, J. J. (1982). Neural networks and physical systems with emergent collective computational abilities. Proceedings of the $\mathrm{Na}$ tional Academy of Sciences, 79, 2554-2558.

Hughes, H. C., Layton, W. M., Baird, J. C., \& Lester, L. S. (1984). Global precedence in visual pattern recognition. Perception \& Psychophysics, 35, 361-371.

Kahneman, D., \& Henik, A. (1981). Perceptual organization and attention. In M. Kubovy \& J. R. Pomerantz (Eds.), Perceptual organization (pp. 181-211). Hillsdale, NJ: Erlbaum.

Kahneman, D., \& Treisman, A. (1984). Changing views of attention and automaticity. In R. Parasuraman \& D. R. Davies (Eds.), Varieties of attention (pp. 29-62). New York: Academic Press.

KoEHLER, W. (1928). An aspect of Gestalt psychology. In C. Murchison (Ed.), Psychologies of 1925 (pp. 163-195). Worcester, MA: Clark University Press.

Kramer, A., \& Watson, S. (1996). Object-based visual selection and the principle of uniform connectedness. In A. Kramer, M. Coles, \& G. Logan (Eds.), Converging operations in the study of visual selective attention (pp. 395-414). Washington, DC: American Psychological Association.

KuRYLO, D. D. (1997). Time course of perceptual grouping. Perception \& Psychophysics, 59, 142-147.

Luna, D., Marcos-Ruiz, R., \& Merino, J. M. (1995). Selective attention to global and local information: Effects of visual angle, exposure duration, and eccentricity on processing dominance. Visual Cognition, 2, 183-200

Mack, A., Tang, B., Tuma, R., Kahn, S., \& Rock, I. (1992). Perceptual organization and attention. Cognitive Psychology, 24, 475-501.

MARR, D. (1982). Vision. San Franciso: W. H. Freeman.

MiLLER, J. (1981). Global precedence in attention and decision. Journal of Experimental Psychology: Human Perception \& Performance, 7, 1161-1174.
MoORE, C. M., \& EGETH, H. (1997). Perception without attention: Evidence of grouping under conditions of inattention. Journal of Experimental Psychology: Human Perception \& Performance, 23, 339352.

Navon, D. (1977). Forest before trees: The precedence of global features in visual perception. Cognitive Psychology, 9, 353-383.

Navon, D., \& NormaN, J. (1983). Does global precedence really depend on visual angle? Journal of Experimental Psychology: Human Perception \& Performance, 9, 955-965.

NeIsser, U. (1967). Cognitive psychology. Englewood Cliffs, NJ: Prentice-Hall.

Palmer, S. (1992). Common region: A new principle of perceptual grouping. Cognitive Psychology, 24, 436-447.

.PALMER, S., \& Rock, I. (1994). Rethinking perceptual organization: The role of uniform connectedness. Psychonomic Bulletin \& Review, 1, 29-55.

Pomerantz, J. R. (1981). Perceptual organization in information processing. In M. Kubovy \& J. R. Pomerantz (Eds.), Perceptual organization (pp. 141-180). Hillsdale, NJ: Erlbaum.

Pomerantz, J. R. (1983). Global and local precedence: Selection attention in form and motion perception. Journal of Experimental Psychology: General, 112, 516-540.

Rock, I. (1986). The description and analysis of object and event perception. In K. R. Boff, L. Kaufman, \& J. P. Thomas (Eds.), Handbook of perception and human performance (chap. 33, pp. 1-46). New York: Wiley.

Treisman, A. (1986). Properties, parts, and objects. In K. R. Boff, L. Kaufman, \& J. P. Thomas (Eds.), Handbook of perception and human performance (chap. 35, pp. 1-46). New York: Wiley.

WATSON, S. E., \& KRAMER, A. F. (1999). Object-based visual selective attention and perceptual organization. Perception \& Psychophysics, 61, 31-49

WERTHEIMER, M. (1923). Untersuchungen zur Lehre von der Gestalt: 11 Psychologische Forschung, 4, 301-350. [Partial translation in W. D. Ellis (Ed.) (1950). A sourcebook of Gestalt psychology (pp. 71-81). New York: Humanities Press.]

ZEEMAN, E. C. (1962). The topology of the brain and visual perception. In M. K. Fort (Ed.), The topology of 3-manifolds (pp. 240-256). Englewood Cliffs, NJ: Prentice-Hall.

\section{NOTE}

1. Though a figure-ground segmentation process may also be needed for the proximity conditions, the time costs involved may be assumed to be minimal.

\section{APPENDIX \\ Supplementary Experiment}

The purpose of this experiment was to examine whether the lines connecting local circles were slower to be perceived, relative to the stimuli formed by proximity grouping in Experiment 1. Two sets of stimuli were used. Stimuli in Set A were the same as those used in the proximity grouping condition in Experiment 1. Stimuli in Set B were composed of target letters made up of only the lines that connected the circles of Stimulus Set B in Experiment 1. Measurement of the subjects' RTs to the two sets of stimuli made it possible to compare relative speeds with which the lines and circles in the target letters were perceived.

\section{Method}

Subjects. The subjects were 14 young adults ( 3 female, 11 male; $20-35$ years of age). All subjects were right-handed and had normal or corrected-to-normal vision.

Apparatus, Stimuli, and Procedure. All aspects were the same as those used in Experiment 1, with the following exceptions: Stimulus Set A was the same as Set A in Experiment 1. Stimulus Set B was formed by removing the local circles from Stimulus Set B in Experiment I; thus, the target letters were made up of the connecting lines only. There were two ISI conditions (either 16 or $144 \mathrm{msec}$ ). After 16 trials for practice, each subject performed 96 experimental trials. 
Table A1

Reaction Times (RTs; in Milliseconds) and Error Rates for the Two Sets of Stimuli in the Supplemental Experiment

\begin{tabular}{cccccc}
\hline & \multicolumn{2}{c}{ Stimulus Set A } & & \multicolumn{2}{c}{ Stimulus Set B } \\
\cline { 2 - 3 } \cline { 5 - 6 } ISI (msec) & RT (msec) & Error $(\%)$ & & RT (msec) & Error $(\%)$ \\
\hline 16 & 440 & 3.6 & & 433 & 1.8 \\
144 & 439 & 3.2 & & 436 & 3.7 \\
\hline
\end{tabular}

\section{Results and Discussion}

Table Al shows the mean error rates and RTs for the two sets of stimuli. ANOVAs showed that, for both error rates and RTs, neither the main effects of stimulus type and ISI nor their interaction were significant ( $p>0.2$, for all analyses).

The data indicate that the connecting lines can be perceived as rapidly as the local circles in the proximity grouping condition. The results thus lend little support to the argument that the lines connecting the local circles in the earlier experiments were slow to be perceived and so could not have affected proximity grouping.

(Manuscript received October 24, 1996; revision accepted for publication April 21, 1998.) 